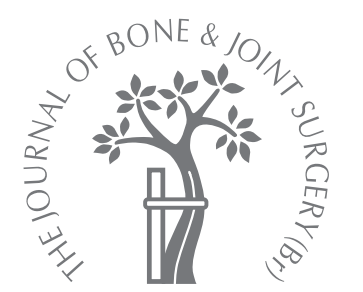

\title{
Rates of fracture in participants and non- participants in the Osteoporosis Prospective Risk Assessment Study
}

P. Gerdhem, K. Åkesson

From Malmö

University Hospital, Sweden
P. Gerdhem, MD, PhD, Orthopaedic Surgeon, Associate Professor Department of Orthopaedics Karolinska University Hospital Huddinge, Karolinska Institute, SE-14186 Stockholm, Sweden.

- K. Åkesson, MD, PhD, Orthopaedic Surgeon, Professor

Department of Orthopaedics Malmö University Hospital, Lund University, SE-20502 Malmö, Sweden.

Correspondence should be sent to Associate Professor

P. Gerdhem; e-mail: paul.gerdhem@med.lu.se

(c)2007 British Editorial Society of Bone and Joint Surgery doi:10.1302/0301-620X.89B12. $18946 \$ 2.00$

$J$ Bone Joint Surg [Br] 2007;89-B:1627-31.

Received 23 November 2007; Accepted after revision 3 July 2007

We invited 1604 randomly selected women, all 75 years of age, to participate in a study on the risk factors for fracture. The women were divided into three groups consisting of 1044 $(65 \%)$ who attended the complete study, $308(19 \%)$ respondents to the study questionnaire only and $252(16 \%)$ who did not respond. The occurrence of the life-time fracture was ascertained from radiological records in all groups and by questionnaires from the attendees and respondents.

According to the radiological records, fewer of the questionnaire respondents ( 88 of 308 , $28.6 \%$ ) and non-respondents $(68$ of $252,27 \%)$ had sustained at least one fracture when compared with the attendees (435 of 1044, 41.7\%; chi-squared test, $p<0.001$ ). According to the questionnaire, fewer of the respondents $(96$ of $308,31.1 \%)$ had sustained at least one previous fracture when compared with the attendees (457 of 1044, 43.7\%; chi-squared test, $p<0.001$ ).

Any study concerning the risk of fracture may attract those with experience of a fracture which explains the higher previous life-time incidence among the attendees. This factor may cause bias in epidemiological studies.

In the Western hemisphere, osteoporosis and associated fractures are an increasing problem and of considerable cost to society. ${ }^{1}$ Valuable information for the identification of risk factors is provided by population-based or casecontrol studies. When performing clinical or epidemiological studies, background information is often obtained from questionnaires. The reliability of the information, however, is rarely verified objectively since such information may have been difficult or impossible to obtain, particularly regarding conditions over a lifetime. Another potential source of bias is related to the rate of response and the representativity of those responding, declining to respond or not answering. With a low rate of response there is always uncertainty as to whether the conclusions can be assumed to hold true for the entire population. The correct identification of fractures is essential in research on osteoporosis and risk assessment since a previous fracture is one of the strongest predictors of a new fracture. ${ }^{2}$ Our aim was to examine the difference in the life-time rate of fracture among participants and non-participants in a population-based study of elderly women, and to compare data on the occurrence of life-time fracture from radiological files and a selfassessment questionnaire.

\section{Patients and Methods}

The Osteoporosis Prospective Risk Assessment study $^{3,4}$ involved 1604 women, aged 75 years, randomly selected from the population files of the city of Malmö. Invitations were sent between December 1995 and May 1999, one week after each woman's 75th birthday. The data in our study refer to three groups of women: 1) 1044 $(65 \%)$ who attended the study ${ }^{3,4}$ with complete participation in the examinations for risk factors on osteoporosis and fracture (attendees); 2) 308 (19\%) who responded to the questionnaire but did not attend the examination (questionnaire respondents); and 3) 252 (16\%) who neither responded nor attended (non-respondents). Among the questionnaire respondents and non-respondents, $152(27 \%)$ gave illness and $376(67 \%)$ unwillingness as reasons for nonparticipation. Despite several attempts, 32 women $(6 \%)$ were among the non-attendees uncontactable. All parts of the study were approved by the ethics committee of Lund University.

Fracture data. The radiological registers of Malmö University Hospital were searched for fractures occurring at any time during life in all 1604 women. There is only one hospital in the city with a radiological department. This department is unique in that it has kept all 
Table I. Data from the radiological archives concerning the life-time occurrence of fracture up to the age of 75 years for all 1604 women in the Osteoporosis Prospective Risk Assessment study. ${ }^{3,4}$ Comparisons are made between the 1044 study attendees, the 308 questionnaire respondents and the 252 non-respondents

\begin{tabular}{llllr}
\hline & Attendees & Respondents & Non-respondents & Nolue \\
\cline { 2 - 4 } Type of fracture & Number (\%) & Number (\%) & Number (\%) & $\begin{array}{l}\text { p-value } \\
\text { (chi-squared test) }\end{array}$ \\
\hline Any & $435(41.7)$ & $88(28.6)$ & $68(27.0)$ & $<0.001$ \\
Hip & $50(4.8)$ & $7(2.3)$ & $7(2.8)$ & 0.079 \\
Distal forearm & $173(16.6)$ & $41(13.3)$ & $28(11.1)$ & 0.059 \\
Vertebral & $57(5.5)$ & $11(3.6)$ & $9(3.6)$ & 0.241 \\
Proximal humerus & $43(4.1)$ & $14(4.5)$ & $7(2.8)$ & 0.533 \\
Pelvic & $15(1.4)$ & $61(19.8)$ & $2(0.8)$ & $0.251^{*}$ \\
Any osteoporotic & $280(26.8)$ & & $48(19.0)$ & 0.005 \\
\hline
\end{tabular}

* Fisher's exact test

Table II. Frequency of fractures up to the age of 75 years for the 1044 study attendees, 308 questionnaire respondents and 252 non-respondents. Data is given for fractures found in the radiological archive and for fracture data from the self-assessment questionnaire

\begin{tabular}{|c|c|c|c|c|c|}
\hline \multirow[b]{3}{*}{ Number of fractures } & \multicolumn{3}{|c|}{ Data from the radiological archive } & \multicolumn{2}{|c|}{ Data from questionnaire } \\
\hline & Attendees & Respondents & Non-respondents & Attendees & Respondents \\
\hline & Number (\%) & Number $(\%)$ & Number (\%) & Number (\%) & Number (\%) \\
\hline 0 & $609(58.4)$ & $220(71.5)$ & $184(73.0)$ & $587(56.2)$ & $212(68.8)$ \\
\hline 1 & $277(26.5)$ & $60(19.5)$ & $41(16.3)$ & $285(27.3)$ & $76(24.7)$ \\
\hline 2 & $95(9.1)$ & $20(6.5)$ & $18(7.1)$ & $111(10.6)$ & $15(4.9)$ \\
\hline 3 & $36(3.4)$ & $4(1.3)$ & $7(2.8)$ & $35(3.4)$ & $4(1.3)$ \\
\hline 4 & $18(1.7)$ & $2(0.6)$ & $2(0.8)$ & $23(2.2)$ & $1(0.3)$ \\
\hline 5 & $4(0.4)$ & $1(0.3)$ & $0(0)$ & $1(0.1)$ & $0(0)$ \\
\hline 6 & $2(0.2)$ & $0(0)$ & $0(0)$ & $2(0.2)$ & $0(0)$ \\
\hline 7 & $2(0.2)$ & $0(0)$ & $0(0)$ & $0(0)$ & $0(0)$ \\
\hline 8 & $1(0.1)$ & $1(0.3)$ & $0(0)$ & $0(0)$ & $0(0)$ \\
\hline
\end{tabular}

radiographs and their reports since the beginning of the 1900s. Radiography can be performed in two smaller outpatient units in the city. They have no facilities for the care of fractures and therefore most patients are referred to the hospital where the radiographs are registered. The proportion of patients diagnosed and treated for fractures in the city and not registered at our hospital has previously been estimated to be less than $3 \% .^{5}$

Every resident in Sweden has a unique ten-digit personal ID number based on their date of birth and this is always used when in contact with public services such as health care. Based on the radiological records, any previous fracture and the age at injury were registered retrospectively for each participating woman. Fractures reported by the attendees which could not be found in the radiological records were checked for in the files of the orthopaedic department. If a participant recorded a fracture as occurring at a different hospital, no further verification was made.

A self-assessment questionnaire, checked for completeness by the research personnel, was used to register any previous fracture, including type, location and age at fracture for the 1044 attendees and 308 questionnaire respondents.

Statistical analysis. The chi-squared test was used when the proportion of women with at least one fracture in the different groups was compared. If expected or observed cell counts were five or less, Fisher's exact test was used. Differences in the rates of fracture were calculated not only for the type of fracture but also for specific sites, such as the hip, distal forearm, vertebra, proximal humerus and pelvis. These specific fractures were also grouped as 'typical osteoporotic'. The Kruskal-Wallis analysis of variance (ANOVA) test was used when the number of fractures per person in three different groups was compared and the Mann-Whitney U test when the number of fractures per person in two different groups was assessed. A p-value $<0.05$ was regarded as being statistically significant.

\section{Results}

According to the radiological records, 591 of the 1604 women $(36.8 \%)$ had sustained at least one fracture in their first 75 years. There was a significant difference between the three groups concerning any type of fracture and typical osteoporotic fractures (Table I). Subsequent tests in the groups showed that fewer of the questionnaire respondents ( 88 of $308,28.6 \%$ ) and non-respondents (68 of $252,27 \%$ ) had sustained at least one fracture when compared with the attendees (435 of 1044, 41.7\%) (chi-squared test, $\mathrm{p}<0.001$ respectively). Tests between the groups showed that fewer of the questionnaire respondents (61 of 308, 19.8\%) and nonrespondents (48 of $252,19 \%$ ) had sustained at least one 


\begin{tabular}{|c|c|c|c|}
\hline \multirow[b]{2}{*}{ Type of fracture } & \multirow{2}{*}{$\begin{array}{l}\text { Attendees } \\
\text { Number (\%) }\end{array}$} & \multirow{2}{*}{$\begin{array}{l}\text { Respondents } \\
\text { Number (\%) }\end{array}$} & \multirow[b]{2}{*}{$\begin{array}{l}\text { p-value } \\
\text { (chi-squared test) }\end{array}$} \\
\hline & & & \\
\hline Any & $457(43.7)$ & 96 (31.1) & $<0.001$ \\
\hline Hip & $56(5.4)$ & $11(3.6)$ & 0.203 \\
\hline Distal forearm & $198(18.9)$ & $32(10.4)$ & $<0.001$ \\
\hline Vertebral & $25(2.4)$ & $2(0.6)$ & $0.062^{*}$ \\
\hline Proximal humerus & $36(3.4)$ & $4(1.2)$ & $0.055^{*}$ \\
\hline Pelvic & $18(1.7)$ & $3(0.9)$ & $0.441^{*}$ \\
\hline Any osteoporotic & $286(27.4)$ & 47 (15.3) & $<0.001$ \\
\hline
\end{tabular}

* Fisher's exact test

typical osteoporotic fracture when compared with the study attendees (280 of $1044,26.8 \%$; chi-squared test, $\mathrm{p}=0.013$ and $\mathrm{p}=0.011$, respectively).

The number of fractures per person was compared in the three groups (Table II) and there were significant differences among them (Kruskal-Wallis test, ANOVA, $\mathrm{p}<0.001$ ). Subsequent tests of the groups showed that the 1044 attendees had sustained more fractures per person (median; 0, 75\% percentile 1; 95\% percentile 3; Mann-Whitney U test, $\mathrm{p}<0.001$ ) than the 308 questionnaire respondents (median; $0,75 \%$ percentile $1 ; 95 \%$ percentile 2 ). The attendees had also sustained more fractures per person (Mann-Whitney U test, $\mathrm{p}<0.001$ ) than the non-respondents (median; 0, 75\% percentile $1 ; 95 \%$ percentile 2 ).

According to the questionnaires, 553 of the 1352 attendees and respondents $(41 \%)$ had sustained at least one previous fracture. Significantly fewer of the questionnaire respondents (96 of $308,31.1 \%$ ) had sustained at least one previous fracture when compared with the study attendees (457 of $1044,43.7 \%$, Table III) (chi-squared test, $\mathrm{p}<0.001)$. Regarding specific types of fracture, this was significant for distal forearm fractures and any osteoporotic fracture (Table III).

Fractures recorded by questionnaire in 1044 attendees which could not be verified in the radiological archives were also searched for in the orthopaedic files of our hospital. This verified another 44 fractures in 36 women. We also found that five 'fractures' in the questionnaire were likely to be misnomers (one injury of the knee, one injury of the wrist, one dislocation of the shoulder, one case of elbow surgery and one of epicondylitis). Of the 1044 attendees, 74 recorded that the treatment of 82 fractures had been undertaken outside our hospital. However, 36 of these fractures were registered in our radiological or orthopaedic files.

By comparing the radiological registration of age and type of fracture with the questionnaire data, the methods for fracture confirmation could be combined for the 1044 attendees and 308 questionnaire respondents. After comparison of those fractures found in the radiological archives and remembered by an individual and the subsequent

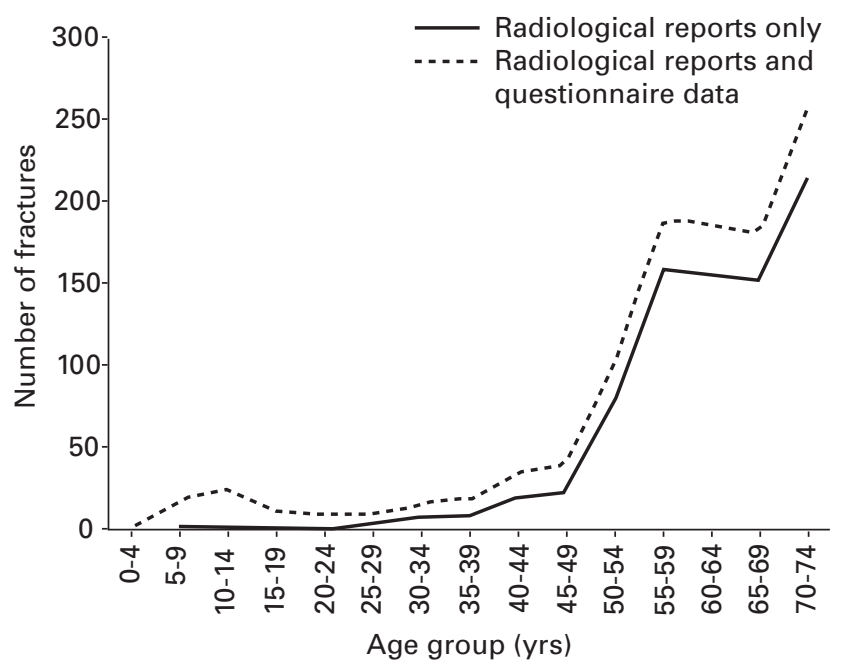

Fig. 1

Graph showing fracture confirmation from the radiological files alone, and the radiological files and questionnaire data combined for the study attendees and the questionnaire respondents $(n=1352)$. A discrepancy between fractures found in the radiological files and questionnaire data was seen in youth when relatively few fractures occur. The incidence rose steeply after the age of 50 years and another increase was seen after the age of 70 years.

exclusion of duplicates, the percentage of women who sustained at least one fracture was $49 \%$ (661 of 1352). A discrepancy between fractures in the archive and recall data was seen especially in youth when relatively few fractures occurred (Fig. 1). The incidence of fracture rose steeply after the age of 50 years and another increase was seen after the age of 70 years (Fig. 1).

During their life-times, the 1352 women sustained 67 hip, 325 distal forearm, 98 vertebral and 70 proximal humeral fractures. No hip fractures occurred before the age of 40 years and their frequency increased after 55 years, with a maximum in the group aged 70 to 74 years. Distal forearm fractures were seen at all ages, with a maximum in the group aged 55 to 59 years. All vertebral fractures were 
Table IV. Data for women with at least one fracture in the radiological archive in comparison to questionnaire recall data for the 1352 women who responded to the questionnaire

\begin{tabular}{lcc}
\hline Type of fracture & $\begin{array}{l}\text { Number of fractures in } \\
\text { radiological archive }\end{array}$ & $\begin{array}{l}\text { Number of women } \\
\text { recalling fracture (\%) }\end{array}$ \\
\hline Any & 523 & $410(78.4)$ \\
Hip & 57 & $53(93.0)$ \\
Distal forearm & 214 & $155(72.4)$ \\
Vertebral & 68 & $17(25.0)$ \\
Proximal humerus & 57 & $22(38.6)$ \\
Pelvic & 16 & $9(56.3)$ \\
Any osteoporotic & 341 & $239(70.1)$ \\
& & $117(77.5)$ \\
Any fracture $<50$ years & 151 & $353(78.3)$ \\
Any fracture $>50$ years & 451 &
\end{tabular}

sustained after the age of 40 years, with a maximum incidence in the group aged 70 to 74 years. Proximal humeral fractures were seen after the age of 45 years, with a maximum incidence in the group aged 70 to 74 years.

Of the 1352 women who responded to the questionnaire, $523(38.6 \%)$ had at least one fracture registered in the radiological archives. According to the questionnaire, $78.4 \%$ (410) of the 523 women recalled sustaining a fracture (Table IV). Hip and distal forearm fractures were more often correctly identified than vertebral fractures, which were remembered only by $25 \%$ (17 of 68 ) of the women (Table IV). The proportion of women remembering at least one fracture before and after the age of 50 years was similar (77.5\% (117 of 151$)$ vs $78.3 \%$ (353 of 451$)$ ) (Table IV).

For the 1352 women, the sensitivity for finding a fracture in the radiological files when a woman recalled a fracture in the questionnaire was $74 \%$ (95\% confidence interval (CI) 72 to 76). The specificity (no fracture found in the radiological files when a woman did not recall a fracture) was $86 \%$ (95\% CI 84 to 88 ).

\section{Discussion}

Our study has shown that studies on osteoporosis and fractures may attract individuals who have sustained fractures, that individuals tend to under-report previous fractures and that data from a combination of different sources may approach true life-time incidence of fracture.

Our study has several advantages. The cohort is well defined, there are detailed fracture data including information from non-participants, and the study covers the entire life up to the age of 75 years. There are several possible limitations. The reason for the discrepancy between the different sources of information may be that some fractures were sustained outside our catchment area and the patients did not attend our hospital, or that in some fractures for example ribs and toes, either radiography was not carried out or was performed at one of the two private outpatient clinics and, when no specific treatment was needed, not referred to our hospital. Furthermore, during a period of 75 years we cannot exclude the possibility that some radiological files were lost.
Up until 1989, the studies assessing agreement between questionnaire data and corresponding data from various medical files, did not assess fractures. After that time a few such studies were performed. ${ }^{5,6-16}$

Certain fractures are more commonly recalled, such as in the forearm and those which occurred early in life. ${ }^{13,14,16}$ We found no difference in the extent of under-reporting between attendees and questionnaire respondents. Verification of fractures from hospital files revealed a dissimilar pattern among attendees and respondents, suggesting that the former had a significantly higher prevalence.

It is often a concern that respondents in randomlyselected studies are not representative of the entire population and, for example, may include the healthier members of the population. However, condition-related studies may attract a greater number of those with the disease or condition. In order to address selection bias and its impact on the pattern of fracture, we were able to obtain the questionnaire from $55 \%$ (308 of 560) of the women unwilling to attend the full investigation. We were also able to obtain information on the reason for not attending in 94\% (528 of 560) of the non-attendees. Fractures were registered for the entire cohort (1604 women). If those who did not attend the complete examination represented a less healthy group we would have expected a higher number of fractures. However, we found the opposite. This finding is supported by another study in women in which participants had more fractures than non-participants. ${ }^{15}$

Peripheral fractures are normally identified easily while vertebral fractures are not. We decided to register only the fracture of a particular vertebra in a person once. Although this was done to avoid multiple counting, our approach may have underestimated worsening of vertebral compression fractures. However, specific grading of vertebral fractures was not our purpose. Certain types, such as fissure or hairline fractures, may not always be perceived as a fracture, which may also account for differences in the prevalence between radiological files and self-assessment questionnaires. Another difficulty when using self-assessment is the possibility of misclassifying the fracture region. One 
approach to this problem may be the use of a body manikin ${ }^{14}$ but this is not necessarily more accurate.

Self-reporting of fractures is often used in studies, but it may be difficult to estimate its accuracy, particularly when dealing with long-term or lifetime reporting. There may also be a tendency to forget with increasing age. However, the proportion of women remembering at least one fracture did not differ before and after the age of 50 years. We have previously shown that up to $30 \%$ of 60 - to 80 -year old women who had a fracture of the wrist had forgotten the fracture or on which side it had occurred, ${ }^{10}$ and in a study of 391 participants Jonsson et $\mathrm{al}^{5}$ found that $40 \%$ of the fractures had been forgotten. ${ }^{5}$

We did not test the validity of the questionnaire by asking the women to repeat it. Instead, we tested reliability by registering fractures from the hospital files. Women appear to remember fractures in childhood and youth as well as recent fractures (Fig. 1). Gross under-reporting of vertebral fractures is not surprising since they may not be described as a fracture and are therefore difficult to differentiate from back pain. For other fractures, particularly those not requiring specific treatment, it may be very difficult for the patient to differentiate between fracture and soft-tissue injury. Fractures of the hip and distal forearm seem to be easier to remember than other types. ${ }^{17,18}$

The use of questionnaires alone for confirmation of a fracture may underestimate their true life-time occurrence by $22 \%$ (any type of fracture) and 30\% (any osteoporotic fracture), as seen in our study when questionnaire data were compared and combined with radiological data. While the true life-time incidence of fracture may be very difficult to obtain, we believe the use of a combination of sources may give this. In the analysis of specific types of fracture, the age-specific patterns agreed with other reports. ${ }^{19}$

Self-reporting of fracture underestimated their number, especially those occurring in middle age. Memory recall was similar in attendees and questionnaire respondents. There was no significant difference in reliability between them, and willingness to participate did not seem to affect the result. We found the sensitivity for having one or more fractures to be $74 \%$ for the attendees and respondents. This is slightly lower than in similar reports, probably because it is a 75-year retrospective study. Most other studies are shorter and prospective, with sensitivity for any fracture ranging between $78 \%$ and $88 \%$ and specificity $89 \%$ and $96 \% .^{11,13,14,16}$

In summary, between $36.8 \%$ (591 of 1604) and 49\% (661 of 1352) of 75-year old women in Malmö experienced a fracture during their lifetime. The rate of fracture varies depending on the source used for fracture assessment (radiological data only or radiological data com- bined with questionnaire data). A study of a specific disease, in this case osteoporosis and fracture, may attract those with the disease or risk factors. This may explain the higher previous life-time incidence of fracture in the attendees of the Osteoporosis Prospective Risk Assessment study. ${ }^{3,4}$ Since this factor may inflict bias in similar epidemiological studies, fracture confirmation is likely to be better when data are retrieved from several sources.

The authors would like to acknowledge Apotekare Hedberg's Foundation for Medical Research and the Alfred Österlund Foundation for financial support. No benefits in any form have been received or will be received from a commercial party related directly or indirectly to the subject of this article.

\section{References}

1. Melton LJ 3rd. Adverse outcomes of osteoporotic fractures in the general population. J Bone Miner Res 2003;18:1139-41.

2. Kanis JA. Diagnosis of osteoporosis and assessment of fracture risk. Lancet 2002;359:1929-36.

3. Gerdhem P, Ringsberg KA, Akesson K, Obrant KJ. Influence of muscle strength, physical activity and weight on bone mass in a population-based sample of 1004 elderly women. Osteoporos Int 2003;14:768-72.

4. Gerdhem P, Ivaska KK, Alataolo SL, et al. Biochemical markers of bone metabolism and prediction of fracture in elderly women. J Bone Miner Res 2004;19:386-93.

5. Jonsson B, Gärdsell P, Johnell 0, Redlund-Johnell I, Sernbo I. Remembering fractures: fracture registration and proband recall in southern Sweden. J Epidemiol Community Health 1994;48:489-90.

6. Harlow SD, Linet MS. Agreement between questionnaire data and medical records: the evidence for accuracy of recall. Am J Epidemio/ 1989;129:233-48.

7. Rees JL. Accuracy of hospital activity analysis data in estimating the incidence of proximal femoral fracture. Br Med J (Clin Res Ed) 1982;284:1856-7.

8. Beard CM, Melton LJ 3rd, Cedel SL, Richelson LS, Riggs BL. Ascertainment of risk factors for osteoporosis: comparison of interview data with medical record review. J Bone Miner Res 1990;5:691-9.

9. van Hemert AM, Vandenbroucke JP, Birkenhager JC, Valkenburg HA. Prediction of osteoporotic fractures in the general population by a fracture risk score: a 9-year follow-up among middle-aged women. Am J Epidemio/ 1990;132:123-35

10. Akesson K, Gardsell P, Sernbo I, Johnell O, Obrant KJ. Earlier wrist fracture: a confounding factor in distal forearm bone screening. Osteoporos Int 1992;2:201-4.

11. Nevitt MC, Cummings SR, Browner WS, et al. The accuracy of self-report of fractures in elderly women: evidence from a prospective study. Am J Epidemiol 1992;135:490-9.

12. Paganini-Hill A, Chao A. Accuracy of recall of hip fracture, heart attack, and cancer: a comparison of postal survey data and medical records. Am J Epidemio/ 1993;138:2101-6.

13. Honkanen K, Honkanen R, Heikkinen L, Kroger H, Saarikoski S. Validity of selfreports of fractures in perimenopausal women. Am J Epidemio/ 1999;150:511-16.

14. Ismail AA, O'Neill TW, Cockerill W, et al. Validity of self-report of fractures: results from a prospective study in men and women across Europe: EPOS Study Group. Osteoporos Int 2000;11:248-54.

15. Hasserius R, Karlsson MK, Nilsson BE, Redlund-Johnell I, Johnell O. Non-participants differ from participants as regards risk factors for vertebral deformities: a source of misinterpretation in the European Vertebral Osteoporosis Study. Acta Orthop Scand 2002;73:451-4.

16. Ivers RQ, Cumming RG, Mitchell P, Peduto AJ. The accuracy of self-reported fractures in older people. J Clin Epidemiol 2002;55:452-7.

17. Chen Z, Kooperberg C, Pettinger MB, et al. Validity of self-report for fractures among a multiethnic cohort of postmenopausal women: results from the Women's Health Initiative observational study and clinical trials. Menopause 2004;11:264-74.

18. Joakimsen RM, Fønnebø V, Søgaard AJ, et al. The Tromsø study: registration of fractures, how good are self-reports, a computerized radiographic register and a discharge register? Osteoporos Int 2001;12:1001-5.

19. Kanis JA, Johnell 0, Oden A, et al. Long-term risk of osteoporotic fracture in Malmö. Osteoporos Int 2000;11:669-74. 